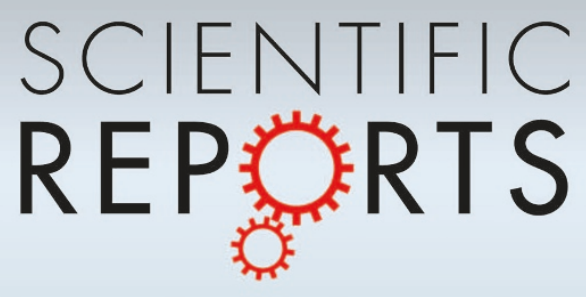

\title{
OPEN A novel signaling pathway regulates colon cancer angiogenesis through Norrin
}

SUBJECT AREAS:

COLORECTAL CANCER

Received

5 March 2014

Accepted

19 June 2014

Published

9 July 2014

Correspondence and requests for materials should be addressed to R.F.H. (Randall. holcombe@mssm.edu)
Kestutis Planutis, Marina Planutiene \& Randall F. Holcombe

Division of Hematology and Medical Oncology, and Tisch Cancer Institute, Icahn School of Medicine at Mount Sinai.

Norrin binds to the frizzled-4 receptor, stimulating canonical Wnt signaling. We investigate here the role of colorectal cancer (CRC) produced Norrin in endothelial cell growth, motility, and blood vessel formation, as well as the expression of the Norrin signaling pathway components in the CRC tumor microenvironment. Norrin conditioned medium produced by CRC cell line $\mathrm{CaCO} 2$ transfected with Norrin expression construct increased endothelial cell motility. Blocking Norrin signaling reduced endothelial cell motility, branch point number $\left(1 / \mathrm{mm}^{2}\right)$, and the network length $\left(\mathrm{mm} / \mathrm{mm}^{2}\right)$ during in vitro angiogenesis. Colorectal tumors express Norrin protein. Endothelial cells in the colorectal tumor microenvironment contain all of the components of the Norrin signaling pathway needed to respond to Norrin protein. This study presents data that Norrin may play a role in the regulation of angiogenesis in the colorectal cancer tumor microenvironment.

M ultiple signaling pathways regulate endothelial cells in the tumor microenvironment. While vascular endothelial growth factors (VEGF) and its family of receptors (VEGFR) are most well defined, there are multiple alternative angiogenic pathways involving fibroblast growth factor (FGF), Notch Delta-like ligand 4 (DLL4) and platelet-derived growth factors (PDGF) ${ }^{1}$. None of these pathways except VEGF are understood enough to allow for FDA approved therapy design.

Wnt signaling is also known to be an important regulator of angiogenesis and of other processes operative in the colorectal cancer tumor microenvironment. Wnt 2 and Wnt5a are over expressed in colorectal tumors and the expression of frizzled $(\mathrm{Fz})$ receptors is tightly regulated by secreted Wnt ligands ${ }^{2,3}$. Wnt signaling contributes to tumor cell proliferation and inhibition of differentiation but is also a critical mediator of endothelial function ${ }^{4,5}$. Wnt3a has been shown to induce endothelial cell proliferation and migration in the context of DVL3 phosphorylation ${ }^{6}$. LEF1, a terminal component of the Wnt signaling cascade, directly influences endothelial cell invasion, and the pathway is basally activated in subconfluent endothelial cells $\mathrm{s}^{7,8}$.

Norrin, a non-Wnt ligand, binds selectively to Fz receptor subtype-4 (Fz4) and induces canonical Wnt signaling in conjunction with cell surface co-receptor LRP5 $5^{9,10}$. This ligand is involved in angiogenesis since overexpression in Norrin deficient mice induces the growth of ocular capillaries ${ }^{11}$ and inactivating mutations results in Norrie disease which is characterized by ocular vascular defects and impaired cochlear vascularization ${ }^{12}$. Abnormalities in Fz4 or LRP5 result in defects in retinal vascular development and in a phenotypically similar condition, familial exudative vitreoretinopathy (FEVR) ${ }^{13}$.

We have previously demonstrated the presence of Norrin, Fz4 and LRP5 mRNA in human tissue biopsies of colon cancer and speculated that Norrin signaling is involved in endothelial cell regulation in the tumor microenvironment ${ }^{14}$. In this study, we demonstrate that Norrin is produced by human colorectal cancer cells, that it directly regulates endothelial cell proliferation and behavior and that all of the critical components necessary to respond to Norrin signals are expressed by endothelial cells in the tumor microenvironment.

\section{Results}

Effect of Norrin on endothelial cells in vitro. Utilizing an in vitro wound healing assay and EAhy926 cells, we demonstrate that Norrin blocking antibody significantly reduces endothelial cell migration, suggesting a basal dependence on Norrin signaling (Figure $1 \mathrm{~A}-\mathrm{C}$ ). The addition of supplemental Norrin promotes endothelial cell migration and motility (Figure $1 \mathrm{D}-\mathrm{F}$ ). In the latter case, Norrin was provided by the addition of conditioned medium from $\mathrm{CaCO} 2$ colon cancer cells following transfection with a Norrin expression construct since this cell line is one of few colon cancer cell lines that does not basally produce Norrin ${ }^{14}$. In an in vitro angiogenesis primary 

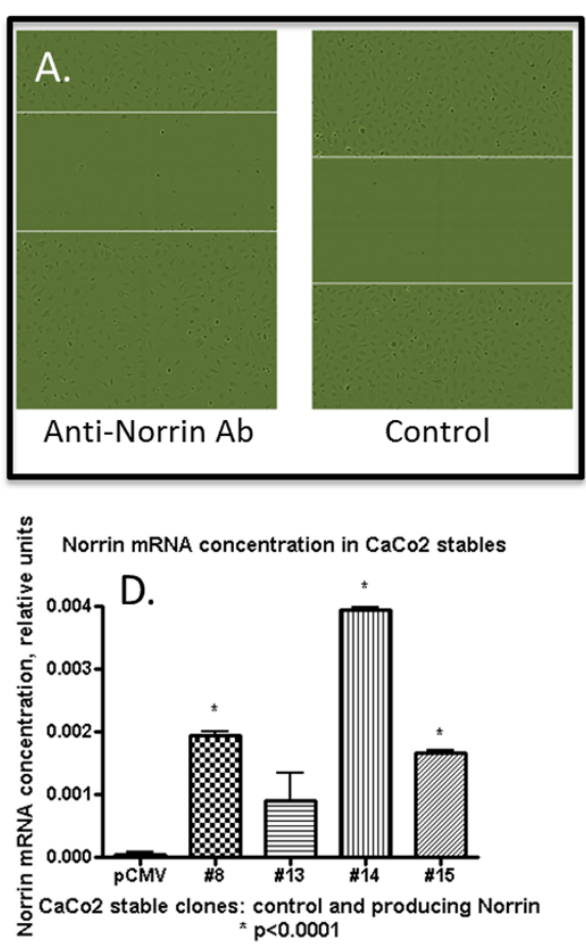
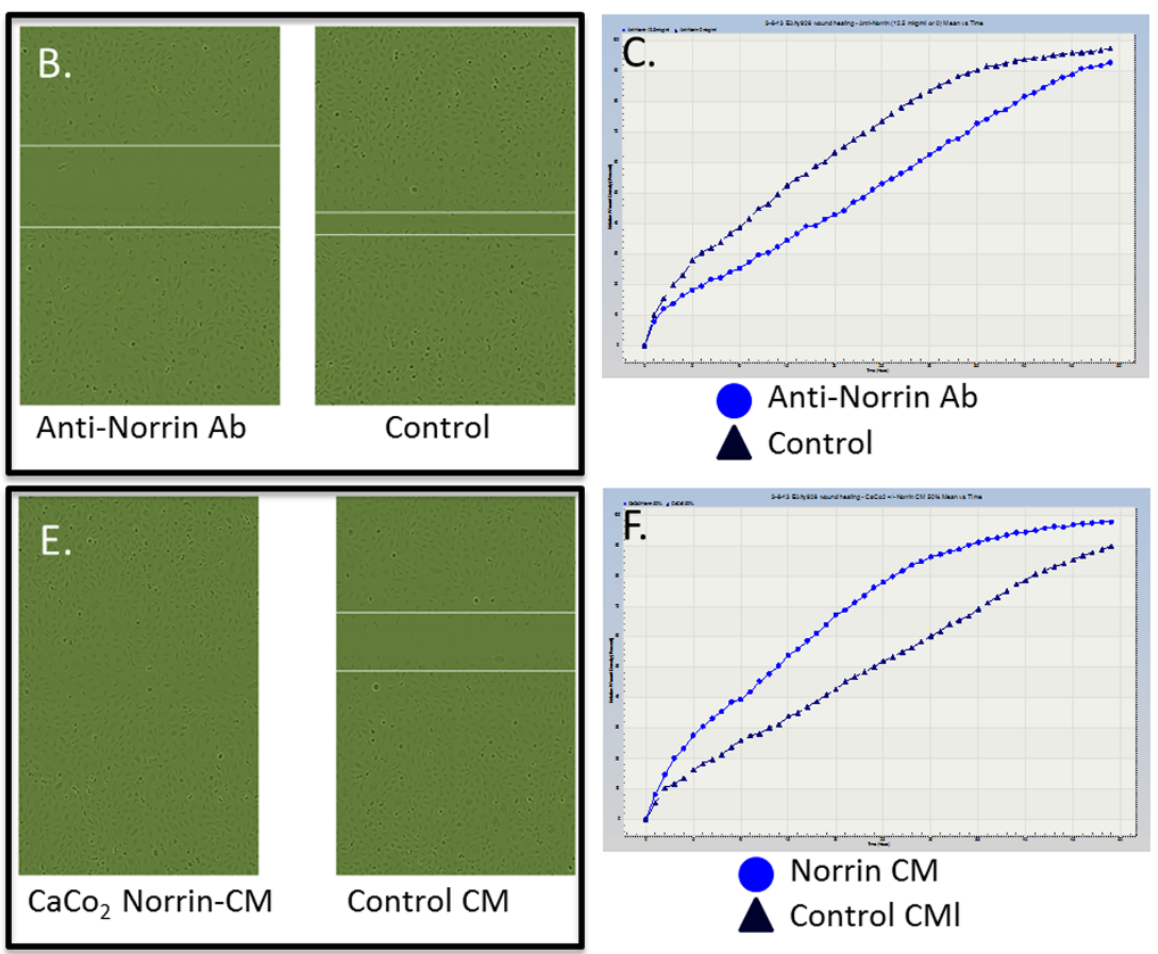

Figure 1 Norrin-containing conditioned medium promotes endothelial cell growth/motility in an EAhy926 endothelial cell wound healing assay; Norrin blocking antibody inhibits wound healing in monolayer of human endothelial cells EAhy926. Panel (A) and Panel (B). Wound healing assay demonstrating growth and migration of EAhy926 endothelial cells at time 0 (panel A) and after 24 hours (Panel B). After 24 hours, Norrin blocking antibody $(12.5 \mathrm{ug} / \mathrm{ml})$ treated EAhy926 endothelial cells migrate shorter distances than control isotype IgG treated cells. Panel (C). The quantitative results of the migrated distance measurement demonstrate graphically that anti-Norrin blocking antibody impedes endothelial cell growth and migration toward the denuded zone. Panel (D). Norrin mRNA is not expressed basally in the CRC cell line CaCO2. Transfection of these cells with a Norrin/pCMV Script expression construct restores the Norrin mRNA expression. Expression levels in several representative stable transfectant clones are depicted. The increased expression above control pCMV-transfected clones is statistically significant $(\mathrm{p}<0.001)$. Panel (E). Wound healing assay demonstrating that, after 30 hours, Norrin conditioned medium-treated EAhy926 endothelial cells migrate a longer distance than EAhy 926 cells treated with control conditioned medium. After 30 hours, no denuded zone remains in the Norrin treated condition. Panel (F). The quantitative results of the experiment in the Panel E is depicted, indicated that Norrin conditioned medium promotes EAhy926 endothelial cell growth and migration.

endothelial cell culture model, exposure to Norrin blocking antibody reduces the branch point number $(1 / \mathrm{mm} 2)$ and reduces the endothelial cell network length $\left(\mathrm{mm} / \mathrm{mm}^{2}\right)$ during in vitro angiogenesis (Figure 2 and supplemental on-line video). These experiments are consistent with findings from reports that, in normal tissues, Norrin directly affects endothelial cell growth and function ${ }^{11,12}$.

Expression of Norrin protein in colorectal cancers and its blood vessels. Previously, we demonstrated that Norrin mRNA is expressed in colorectal tumors and the majority of CRC cell lines ${ }^{14}$. It was shown in 2012 that Norrin is expressed in retina's endothelial cells ${ }^{16}$. The relatively low level of expression of the Norrin protein in most other tissues has made the detection of Norrin expression problematic. However, through the use of our sensitive double signal amplification system (see The Materials and Methods) we have been able to evaluate the expression of this protein in human colorectal tumors. Norrin protein is produced by colorectal tumors (Figure 3), and in the endothelial cells of tumor blood vessels (Figure 4, Row A). By this semi-quantitative technique, it appears that colorectal tumors express similar levels of Norrin protein as tumor blood vessel endothelial cells.

Expression of Norrin signaling pathway components in CRC endothelial cells. In order for endothelial cells in the tumor microenvironment to respond to Norrin, all functional receptor complex components must be expressed. The first component of
Norrin signaling pathway is Frizzled receptor 4 (Fz4). This is the only $\mathrm{Fz}$ receptor to which Norrin protein binds, leading to activation, most prominently, of the canonical Wnt/beta-catenin pathway. Utilizing the same sensitive double signal amplification methodology used to detect Norrin expression, we demonstrate conclusively that CRC endothelial cells express Fz4 protein (Figure 4, row B). We previously demonstrated that the Lrp5 coreceptor was expressed in colorectal tumors, and cell lines, derived from the colorectal tumors ${ }^{14}$. We demonstrate here that this coreceptor is expressed in colorectal tumor blood vessel endothelial cells (Figure 4, row C). A third component necessary for Norrin signaling in colorectal tumor blood vessels is TSPAN12 molecule. TSPAN12 participation in Norrin receptor complex significantly increases Norrin signaling through nuclear beta-catenin, but does not change Wnt ligand-dependent signaling ${ }^{17}$. Thus, it appears that TSPAN12 is a specific Norrin signal amplifier ${ }^{17}$. Using the sensitive double signal amplification methodology, we demonstrate that TSPAN12 protein is expressed in colorectal tumor blood vessel endothelial cells (Figure 4, row D).

\section{Discussion}

Parental primary human umbilical vein endothelial cells (HUVEC) are known to respond to Wnt3a treatment ${ }^{6,18,19}$ and we have previously defined the responsiveness of the endothelial cell line, EAhy926 to Wnt signals 7 . This capability implies that endothelial cells in the tumor microenvironment respond to Wnt signals known to be operative there ${ }^{2}$. While Norrin-associated Wnt signaling has 


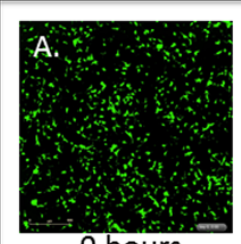

0 hours

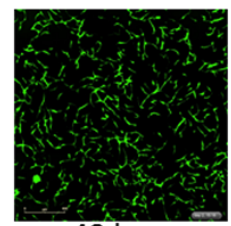

48 hours

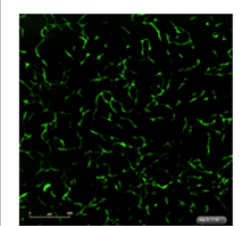

96 hours

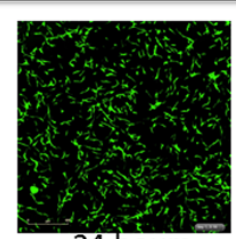

24 hours

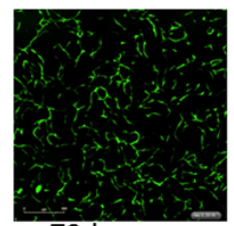

72 hours

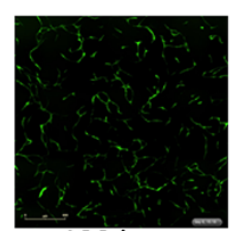

120 hours

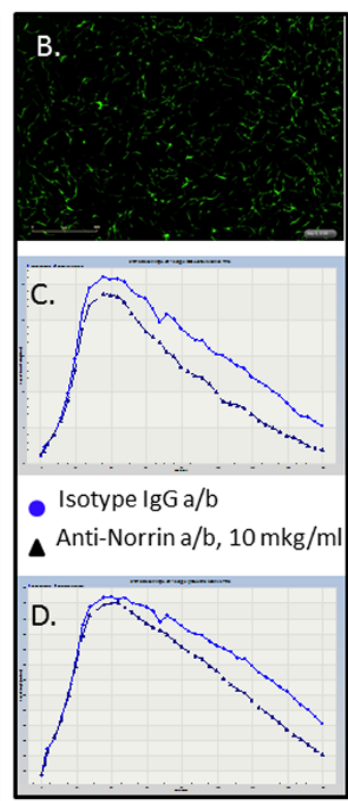

Figure $2 \mid$ Norrin blocking antibody reduces the branch point number (1/ $\left.\mathrm{mm}^{2}\right)$ and the network length $\left(\mathrm{mm} / \mathrm{mm}^{2}\right)$ of endothelial cells during in vitro angiogenesis. Panel (A). Time lapse images of antiogenesis under control contitions from 0 to 120 hours is shown. Green fluorescent protein-stained primary endothelial cells form vessel-like structures that can be maintained up to 120 hours until medium resources are depleted. Panel (B). In vitro angiogenesis at 40 hours in the presence of anti-Norrin antibody, showing markedly reduced vessel formation compared to control. Panel (C). Quantitative representation of the effect of anti-Norrin antibody on branch point number $\left(1 / \mathrm{mm}^{2}\right)$ over 160 hours. Anti-Norrin antibody reduces the branch point number during in vitro angiogenesis. Panel (D). Quantitative representation of the effect of anti-Norrin antibody on network length $\left(\mathrm{mm} / \mathrm{mm}^{2}\right)$ over 160 hours. Anti-Norrin antibody reduces the network length during in vitro angiogenesis.

been linked to angiogenesis in the eye and ear ${ }^{11,12}$, its role in the tumor microenvironment, and specifically in colorectal cancer, has not been previously defined. We detected some time ago the influence of Wnt ligand on the expression of Norrin receptor $\mathrm{Fz}_{4}{ }^{14}$. In this study, we describe that Norrin produced by colorectal tumor and endothelial cells ${ }^{14}$ directly promotes endothelial cell motility and other functions, and that blocking Norrin abrogates this effect. Specifically, blocking the Norrin signal in EAhy926 cells reduces the branch point number and network length $\left(\mathrm{mm} / \mathrm{mm}^{2}\right)$, in vitro measures of angiogenesis. We also show that Norrin is produced in the CRC tumor microenvironment and that all of the cell surface components necessary for Norrin to induce a Wnt signaling cascade, Fz4, Lrp5 and TSPAN12 are expressed on tumor endothelial cells.

Norrin, therefore, may be an additional factor involved in the regulation of angiogenesis in colon cancers. In CRC blood vessels, Wnt/beta-catenin signaling promotes the expression of MMP- $2^{7}$ which hydrolyzes type IV collagen and other connective tissue substrates. During angiogenesis, the basement membrane is degraded by metalloproteinases and other factors to facilitate invasion of endothelial cells through the membrane ${ }^{20}$. Norrin, by activating Wnt signaling, may promote MMP2 production as well as stimulate endothelial cell motility. Norrin and the membrane-associated components of the Norrin receptor complex may be considered as potential new targets for anti-angiogenic therapy. Targeting these proteins, with blocking antibodies for example, may be more effective than attempting to manipulate $\mathrm{Wnt} /$ beta-catenin signaling by targeting intracellular components ${ }^{21}$.
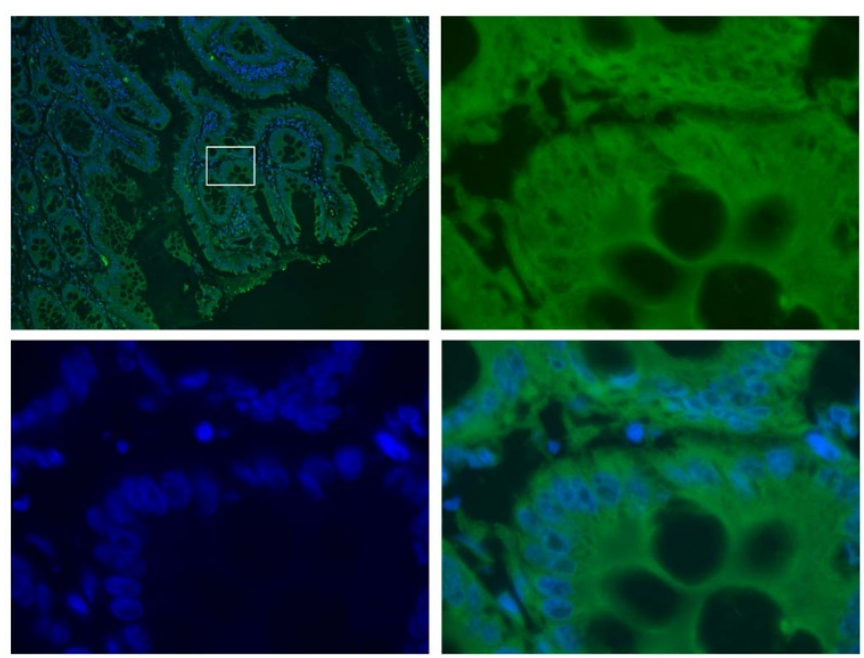

Figure $3 \mid$ Expression of Norrin protein in colorectal tumor epithelial cells. Green fluorescence indicates Norrin. Blue fluorescence indicates nuclei. The microenvironment of the tissue is shown using lower magnification in the top left panel $(5 \times)$. The square demonstrates the region of higher $(100 \times)$ magnification (top right - Norrin staining; bottom left - DAPI; bottom right - merged images).

In conclusion, our data demonstrate that Norrin, is produced by colorectal tumor, regulates endothelial cell motility and in vitro angiogenesis, and is expressed, along with the components of its receptor complex in the colorectal cancer tumor microenvironment. It may play an important role in regulating tumor angiogenesis in vivo in addition to other mediators such as vascular endothelial growth factor. The information produced by this study could be applied in the development of new therapeutic modalities for the colorectal tumors.

\section{Methods}

Constructs and cell lines. The mNorrin gene in pRK5 plasmid (generously provided by Dr. J. Nathans) was subcloned in pCMV Script prior to transfected into a norrin non-expressing colorectal cancer cell line, $\mathrm{CaCO} 2^{14}$. Stable clones were selected, and tested for expression by qRT-PCR. A mixture of norrin mRNA expressing clones was used to produce mNorrin conditioned medium in order to exclude the influence of the cloning artifacts related to the use of separate clones. The human endothelial-like immortalized cell line EaHy926, derived from the fusion of human umbilical vein endothelial cells (HUVEC) with the bronchial carcinoma cell line A549, and expressing an endothelial-like phenotype was a generous gift from Dr. CJ Edgell ${ }^{15}$. It was maintained in DMEM with $4.5 \mathrm{~g} / \mathrm{l}$ glucose and 10\% FBS.

Wound healing assay. The EAhy 926 cells were plated at $50 \%$ confluency and grown overnight. After 24 hours, an innovative device WoundMaker from Essen Bioscience was applied to make a 740 um wound in the monolayer of the endothelial cells. Microscopic images of the wounds were collected every hour during 50 hours to assess the degree of endothelial cell migration into the denuded zone. The images were obtained and analyzed with the IncuCyte FLR System from Essen BioScience. All assays were performed in triplicate under the following conditions: 1) control, 2) addition of anti-Norrin blocking antibody, 3) addition of $\mathrm{CaCO} 2$-produced, Norrincontaining conditioned medium.

Angiogenesis assays. Network length $\left(\mathrm{mm} / \mathrm{mm}^{2}\right)$ (sum of the lengths of all of the networks in the image, normalized by the image area and network branch points (1/ $\mathrm{mm}^{2}$ ) (sum of the branch point count of all of the networks in the image) were estimated using StemKit from Essen BioScience and IncuCyte FLR according to the manufacturer's procedure. (The definitions of the angiogenesis parameters were taken from Essen Bioscience's IncuCyte ZOOM Angiogenesis Processing Overview, page 2). The CellPlayer Angiogenesis StemKit contains human Endothelial Colony Forming Cells (ECFC) and human Adipose Derived Stem Cells (ADSC). Separate, duplicate experiments were conducted each with 8 repeats for each condition. Blocking antibody studies were performed at two different concentrations $(10 \mathrm{ug} / \mathrm{ml}$ and $20 \mathrm{ug} / \mathrm{ml}$ ).

Specific methodology for treatment with blocking antibodies and Norrin conditioned medium. Exposure of the EAhy926 cells to Norrin blocking antibody (R\&D Systems, Cat No. AF 3014) was accomplished according to the manufacturers' 
A.

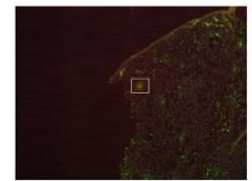

B.

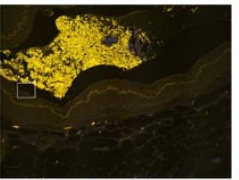

C.

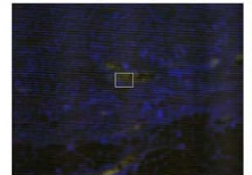

D.

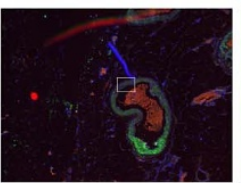

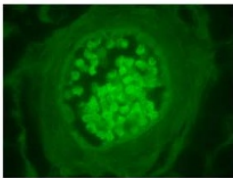
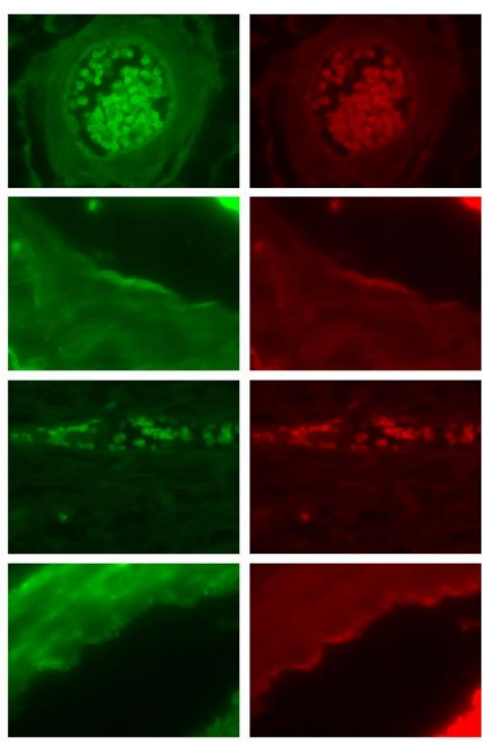
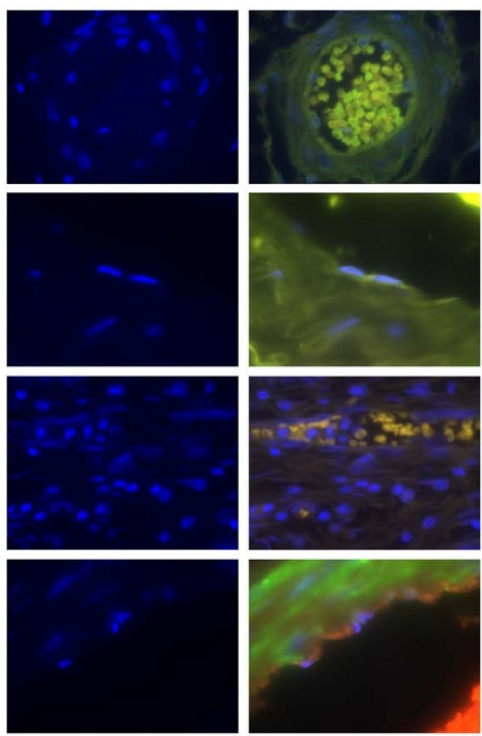

Figure 4 Expression of Norrin signaling components, including Norrin, Frizzled 4, Lrp5 and TSPAN12 in endothelial cells in the CRC tumor microenvironment. In column 1 , low power $(5 \times)$ micrographs of the tumor microenvironment are depicted. The square demonstrates the region of higher $(100 \times)$ magnification (columns 2-5). Green fluorescence (column 2) indicates expression of a specific Norrin signaling component (see row designation, below). Red fluorescence (column 3) marks endothelial cells (anti-CD31 positive) of colorectal tumor blood vessels. Blue fluorescence(column 4) depicts DAPI-stained nuclei. Merged images are depicted in column 5. Row (A). Expression of Norrin in colorectal tumor endothelial cells. Row (B). Expression of Frizzled 4 in colorectal tumor endothelial cells. Row (C). Expression of Lrp5 in colorectal tumor endothelial cells. Row (D). Expression of TSPAN12 in colorectal tumor endothelial cells.

recommendations (The protocol is provided at: http://www.rndsystems.com/ Products/af3014/Applications). The antibody at the final concentration of $12.5 \mathrm{ug} / \mathrm{ml}$ for the wound-healing assay and $10 \mathrm{ug} / \mathrm{ml}$ and $20 \mathrm{ug} / \mathrm{ml}$ for the angiogenesis assay were added directly to the cell culture medium. Cell monitoring began immediately after the addition of the blocking antibody and lasted to the end of the experiment. Matched IgG was included for the controls. Norrin conditioned medium from colorectal cancer cells CaCO2 stably transfected with Norrin/pCMV Script expression construct was prepared using the Nusse lab protocol for Wnt conditioned medium production (described on Wnt Homepage, http://www.stanford.edu/group/ nusselab/cgi-bin/wnt/). CaCo2 cells stably transfected with the Norrin-expression construct were plated at 6-8\% of confluence in DMEM containing 10\% FBS. After 4 days the medium was collected and replaced with the fresh media. After 3 more days the medium was collected again, combined with the first batch, and filtered through 0.2 um filter. This Norrin conditioned medium was diluted $1: 1$ with cell growth medium and used for the treatment of the cells for the wound healing assay. Experiments evaluating the effect of Norrin conditioned medium in the StemKit angiogenesis assay system were not feasible because the concentrations required exceeded levels acceptable in conjunction with the proprietary StemKit medium.

Evaluation of Norrin pathway components in blood vessel endothelial cells by immunofluorescence staining. Fully de-identified colon cancer and normal colonic mucosa tissue slides derived from paraffin-embedded tissue were obtained from archived specimens under a non-human subjects exemption by the institutional review board, in compliance with the Declaration of Helsinki. Samples from 10 individual and distinct patients were available for analysis and immunofluorescence staining. Colorectal tumor slides were triple stained with DAPI (blue) for nuclear DNA, Texas Red (red) for human blood vessel endothelial cells (anti-CD31 mouse monoclonal antibody Abcam (ab 9498) at $1: 10$ dilution, and a secondary antibody conjugate Texas Red-donkey polyclonal against mouse IgG from Abcam (ab 7059) used at $1: 1000$ dilution) and FITC (green) for components of Norrin pathway (goat polyclonal directed against human Norrin (R\&D, AF 3014), diluted till 1:50 from stock $0.2 \mathrm{mg} / \mathrm{ml}$; rabbit polyclonal against human Frizzled 4 (MBL International Corporation, MC-4911), diluted 1:40; goat polyclonal against human Lrp 5 (Abcam, ab 36121), diluted 1:500; rabbit polyclonal against human TSPAN12 (Biorbyt, orb 39384), diluted at $1: 100$. The latter staining utilized a novel sensitive approach by incorporating two signal amplification systems: avidin-biotin conjugates-based from $\mathrm{ABC}$ staining kit (sc-2023 for goat primary antibody and sc- 2018 for rabbit primary antibody, Santa Cruz Biotechnology), and Individual Fluorescein Thyramide Reagent Pack from Perkin-Elmer (SAT701001EA). The main procedural steps were taken from ABC staining kit (Santa Cruz Biotechnology). Shortly, the slides were deparaffinized, fixed with $4 \%$ para-formaldehyde and treated $10 \mathrm{~min}$ at $95^{\circ} \mathrm{C}$ with basic antigen retrieval reagent (R\&D Systems, CTS013). Serum was utilized as a blocking agent to reduce background staining. Slides were incubated with mixture of the two primary antibodies overnight at $4{ }^{\circ} \mathrm{C}$. Incubation with the primary antibody was followed by incubation with biotinylated secondary antibody for Norrin pathway component (final concentration $1 \mu \mathrm{g} / \mathrm{ml}$ ) together with red fluorescent secondary antibody conjugate to reveal the blood vessel endothelial cells, and subsequently with biotinylated horseradish peroxidase-avidin complex. As a substrate for peroxidase we used FluoresceinThyramide System (mentioned above) according to the manufacturer's procedure. Slides were analyzed using epifluorescence confocal microscopy.

Statistical considerations. Expression of Norrin production in individual $\mathrm{CaCO} 2$ clones, in comparison to pCMV transfected control $\mathrm{CaCO} 2$ cells, was performed using a non-paired, two-tailed t-test with level of significance defined as $\mathrm{p}<0.05$. The wound and angiogenesis assays are semi-quantitative and therefore the quantitation graphs were not subjected for formal statistical analysis. Expression of antigens by confocal immunofluorescence was defined by the level of resolution attainable under the described experimental conditions.

1. Ferrara, N. Pathways mediating VEGF-independent tumor angiogenesis. Cytokine \& Growth Factor Rev 21, 21-26, doi:http://dx.doi.org/10.1016/j.cytogfr. 2009.11.003 (2010).

2. Holcombe, R. F. et al. Expression of Wnt ligands and Frizzled receptors in colonic mucosa and in colon carcinoma. J Clin Path-Mol Path 55, 220-226 (2002).

3. Planutis, K., Planutiene, M., Nguyen, A. V., Moyer, M. P. \& Holcombe, R. F. Invasive colon cancer, but not non-invasive adenomas induce a gradient effect of Wnt pathway receptor frizzled $1(\mathrm{Fz} 1)$ expression in the tumor microenvironment. J Transl Med 11, 50, doi:10.1186/1479-5876-11-50 (2013).

4. Giles, R. H., van Es, J. H. \& Clevers, H. Caught up in a Wnt storm: Wnt signaling in cancer. Biochim Et Biophys Acta-Rev Cancer 1653, 1-24, doi:10.1016/s0304419x(03)00005-2 (2003).

5. Dejana, E. The role of wnt signaling in physiological and pathological angiogenesis. Circ Res 107, 943-952, doi:107/8/943 [pii] 10.1161/ CIRCRESAHA.110.223750 (2010).

6. Samarzija, I., Sini, P., Schlange, T., Macdonald, G. \& Hynes, N. E. Wnt3a regulates proliferation and migration of HUVEC via canonical and non-canonical Wnt signaling pathways. Biochem Biophys Res Commun 386, 449-454, doi:S0006291X(09)01175-9 [pii]10.1016/j.bbrc.2009.06.033 [doi] (2009).

7. Planutiene, M., Planutis, K. \& Holcombe, R. F. Lymphoid enhancer-binding factor 1, a representative of vertebrate-specific Lef1/Tcf1 sub-family, is a Wntbeta-catenin pathway target gene in human endothelial cells which regulates matrix metalloproteinase- 2 expression and promotes endothelial cell invasion. Vasc Cell 3, 28, doi:2045-824X-3-28 [pii] 10.1186/2045-824X-3-28 (2011).

8. Goodwin, A. M., Sullivan, K. M. \& D’Amore, P. A. Cultured endothelial cells display endogenous activation of the canonical Wnt signaling pathway and express multiple ligands, receptors, and secreted modulators of Wnt signaling. Dev Dynamics 235, 3110-3120, doi:10.1002/dvdy.20939 (2006).

9. $\mathrm{Xu}, \mathrm{Q}$. et al. Vascular development in the retina and inner ear: control by Norrin and Frizzled-4, a high-affinity ligand-receptor pair. Cell 116, 883-895 (2004). 
10. Clevers, H. Wnt signaling: Ig-norrin the dogma. Curr Biol 14, R436-437, doi:10.1016/j.cub.2004.05.039 (2004).

11. Ohlmann, A. et al. Ectopic norrin induces growth of ocular capillaries and restores normal retinal angiogenesis in Norrie disease mutant mice. J Neurosci 25, 1701-1710, doi:10.1523/JNEUROSCI.4756-04.2005 (2005).

12. Rehm, H. L. et al. Vascular defects and sensorineural deafness in a mouse model of Norrie disease. J Neurosci 22, 4286-4292, doi:2002 (2002).

13. Toomes, C. et al. Mutations in LRP5 or FZD4 underlie the common familial exudative vitreoretinopathy locus on chromosome 11q. Am J Hum Genet 74 721-730, doi:10.1086/383202 (2004).

14. Planutis, K. et al. Regulation of norrin receptor frizzled-4 by Wnt 2 in colonderived cells. BMC Cell Biol 8, Article No., 12 (2007).

15. Edgell, C. J., McDonald, C. C. \& Graham, J. B. Permanent cell-line expressing human factor-VIII-related antigen established by hybridization. Proc Nat Acad Sci 80, 3734-3737 (1983).

16. Lee, H., Jo, D. H., Kim, J. H. \& Kim, J. H. Norrin expression in endothelial cells in the developing mouse retina. Acta Histochemica 115, 447-451, doi:http://dx.doi. org/10.1016/j.acthis.2012.10.008 (2013).

17. Junge, H. J. et al. TSPAN12 Regulates Retinal Vascular Development by Promoting Norrin- but Not Wnt-Induced FZD4/ $\beta$-Catenin Signaling. Cell 139, 299-311, doi:http://dx.doi.org/10.1016/j.cell.2009.07.048 (2009).

18. Goodwin, A. \& D'Amore, P. Wnt signaling in the vasculature. Angiogenesis 5, 1-9 (2002)

19. Wright, M., Aikawa, M., Szeto, W. \& Papkoff, J. Identification of a Wnt-responsive signal transduction pathway in primary endothelial cells. Biochem Biophys Res Commun 263, 384-388 (1999).

20. Davis, G. \& Senger, D. Endothelial extracellular matrix: biosynthesis, remodeling, and functions during vascular morphogenesis and neovessel stabilization. Circ Res 97, 1093-1107 (2005).

21. Najdi, R., Holcombe, R. F. \& Waterman, M. L. Wnt signaling and colon carcinogenesis: beyond APC. J Carcinog 10, 5, doi:10.4103/1477-3163.78111 (2011).

\section{Acknowledgments}

The authors thank Dr. J.Nathans for Norrin gene containing construct, Essen Bioscience for donating the time of IncuCyte FLR system, and Romana Huq, associate director of the shared Research Facilities at the Icahn School of Medicine at Mount Sinai for significant help and microscopy method development.

\section{Author contributions}

R.F.H. and K.P. conceived the study, and had oversight over experimental design and execution. M.P. and K.P. contributed performing tissue expression studies, microscopy, cell based assays, molecular studies, and data analysis. Each author contributed to manuscript preparation and all authors have reviewed and approved the final manuscript.

\section{Additional information}

Supplementary information accompanies this paper at http://www.nature.com/ scientificreports

Competing financial interests: The authors have no competing interests as defined by Nature Publishing Group, or other interests that might be perceived to influence the results and/or discussion reported in this paper.

How to cite this article: Planutis, K., Planutiene, M. \& Holcombe, R.F. A novel signaling pathway regulates colon cancer angiogenesis through Norrin. Sci. Rep. 4, 5630; DOI:10.1038/srep05630 (2014).

This work is licensed under a Creative Commons Attribution-NonCommercialNoDerivs 4.0 International License. The images or other third party material in this article are included in the article's Creative Commons license, unless indicated otherwise in the credit line; if the material is not included under the Creative Commons license, users will need to obtain permission from the license holder in order to reproduce the material. To view a copy of this license, visit http:// creativecommons.org/licenses/by-nc-nd/4.0/ 